\title{
DE LA CIUDADANÍA POLÍTICA A LA CIUDADANÍA SEXUAL. \\ DEBATES POLÍTICOS EN ARGENTINA EN RELACIÓN CON EL CUERPO DE LAS MUJERES
}

\author{
Julieta Evangelina Cano \\ cano.julieta@gmail.com \\ Universidad Nacional de La Plata - Argentina
}

Recibido: 28-02-2016

Aceptado: 26-05-2016

\section{Resumen}

El objetivo de este trabajo es reflexionar sobre la disputa por una ciudadanía plena que aún llevamos adelante las mujeres en Argentina, en donde la lucha por el derecho al aborto todavía es una de las demandas más fuertes del feminismo, frente a un Estado que desoye las voces de los colectivos de mujeres. Iniciamos el trazado del camino que va desde la ciudadanía política a la ciudadanía sexual en la lucha por el sufragio femenino, para luego centrarme en analizar el debate parlamentario que se dio en el año 2006 a propósito de la Ley sobre anticoncepción quirúrgica, para reflexionar sobre los desafíos actuales en relación con el derecho sobre nuestros cuerpos, y una eventual discusión parlamentaria sobre el aborto que todavía es deuda en el Congreso argentino.

Palabras Clave: Ciudadanía, derechos de las mujeres, aborto, cuerpos, anticoncepción quirúrgica, sufragio femenino, ciudadanía sexual.

\footnotetext{
Abstract

The aim of this paper is to reflect on the dispute for full citizenship women still carry on in Argentina, where the struggle for the right to abortion is still one of the strongest demands of feminism, against a State that ignores the voices of women's groups. Initially tracing the path from political citizenship to sexual citizenship in the struggle for women's suffrage, we then focus on analyzing the parliamentary debate that took place in 2006 regarding the law on surgical contraception, to reflect on current challenges in relation to the right over our bodies, and eventual parliamentary debate on abortion.
}

Keywords: Citizenship, women's rights, abortion, bodies, surgical contraception, women's suffrage, sexual citizenship. 


\section{Introducción}

El objetivo de este trabajo es reflexionar sobre la disputa por una ciudadanía plena que aún llevamos adelante las mujeres en Argentina, donde la lucha por el derecho al aborto todavía es una de las demandas más fuertes del feminismo. Frente a un Estado que desoye las voces de las mujeres, de los colectivos de mujeres y de los colectivos feministas en torno a esta deuda pendiente que se enmarca dentro del paradigma de los derechos humanos. Entiendo por ciudadanía sexual aquella que nos permite a las mujeres decidir sobre nuestros propios cuerpos.

Creo que el logro del derecho al aborto se relaciona íntimamente con la ciudadanía femenina, porque se trata de erigirnos como las verdaderas propietarias de nuestros cuerpos, excluyendo al Estado, a la(s) Iglesia(s) y al colectivo de varones de injerencias arbitrarias sobre el mismo. Si la primera propiedad de cada persona es su propio cuerpo, las mujeres nos enfrentamos a un dilema: o bien no somos consideradas personas y nos vemos reducidas a la categoría de objetos, o bien tenemos argumentos sobrados para seguir reclamando que el estatus de seres humanos se nos aplique también a nosotras. Esta lucha no es sólo de las mujeres argentinas, dado que en toda América del Sur, el único país que legisló a favor de la interrupción voluntaria del embarazo fue Uruguay (en el año 2012) además de Guyana.

Para realizar el trazado del camino que va desde la ciudadanía política a la ciudadanía sexual, me abocaré a analizar el debate parlamentario que se dio en el año 2006 a propósito de la sanción de la Ley de anticoncepción quirúrgica. Cabe explicitar que el análisis del debate parlamentario que dio origen a la Ley se realiza a través de los discursos de los/as congresistas y de los/as senadores/as del Parlamento constituido. En la Cámara de Diputados el tratamiento fue el 28 de Junio de 2006 en el Periodo de Sesiones No 124, y en la Cámara de Senadores el tratamiento de la Ley se dio con fecha de 9 de agosto de 2006. Previamente, recorreré los avatares de la consecución del voto para las mujeres en 1947.

Indagar acerca de los argumentos que se desplegaron en el recinto parlamentario con ocasión de tratar un proyecto de Ley que le otorga a las mujeres la posibilidad de decidir acerca de su sexualidad y su reproducción resulta significativo, dado que están íntimamente relacionados con la posibilidad de considerar que las mujeres ya no necesitamos más tutela para decidir sobre nuestros cuerpos y sobre nuestro plan de vida. El análisis del debate parlamentario además responde a una consideración del Derecho como una tecnología de género (Smart, 2000) y a una construcción sexo-genérica que puede o no desarmarse:

"Carol Smart [...] ha observado que la Ley no es simplemente una fuerza coercitiva, sino también es un discurso social poderoso y productivo que crea y refuerza las normas de género. En otras palabras, la Ley no se limita a operar en las realidades de género 
preexistentes, sino que contribuye a la construcción de esas realidades, a menudo en una restricción o una manera perjudicial” (Hunter, McGlynn, Rackley, 2010: 6-7)².

\section{La ciudadanía de las mujeres como problema contemporáneo}

La ciudadanía de las mujeres sigue siendo un problema teórico-práctico contemporáneo, por tres cuestiones: en principio porque el propio concepto está en permanente cambio y definición (Jelin, 1996); en segundo lugar porque como expresa Giordano: "la ciudadanía se pretende universal pero se practica a través de unos derechos que son exclusivos de ciertas categorías sociales" (2012: 65); y por último porque "los actuales estados democráticos han alcanzado un gran nivel de igualdad legal, principalmente a través del mecanismo de la ciudadanía; pero, este mecanismo no parece suficiente para deshacer algunas desigualdades e injusticias que las mujeres sufren, especialmente en los ámbitos cultural, social y económico" (Reverter Bañón, 2008: 33).

Las teorías feministas pusieron en evidencia que, tradicionalmente lo político fue definido como excluyente de todo aquello que no se corresponde con el modelo de ciudadano varón, blanco y burgués. La consigna revolucionaria de 1789 que proclamaba igualdad, libertad y fraternidad claramente excluía a todo el universo femenino: "la modernidad hizo abstracción de la corporeidad y de la biografía del sujeto para convertirlo en ciudadano. Sin embargo, ese desplazamiento encubría un grupo dominante que elaboraba un sujeto prototípico a su imagen: “el hombre", idealmente abstraído, y no la mujer..." (Valobra, 2010: 10).

Una de las críticas más importantes que realizará la teoría feminista sobre las consecuencias de la Revolución paradigmática tendrá que ver con la construcción del individuo de forma androcéntrica:

"La revolución francesa es contradictoria. El universalismo de la declaración de los derechos y deberes del hombre y del ciudadano no concierne verdaderamente a las mujeres: ellas no son individuos. A pesar de ello la revolución les otorga derechos civiles pero ningún derecho político [...]. En todo caso la revolución francesa excluye a las mujeres del ejercicio de la política, empezando por su derecho al voto. Son todas "ciudadanos pasivas" junto con los menores, los extranjeros, los más pobres y los locos" (Perrot, 2008: 180-181).

En Argentina existieron varias voces que reclamaron por los derechos políticos de las mujeres y de hecho el activismo femenino fue crucial para la obtención de los derechos: "ya no es posible atribuir la inequidad legal entre hombres y mujeres a la ausencia o fragilidad de la

\footnotetext{
${ }^{1}$ Traducción propia.
} 
acción colectiva femenina" (Palermo, 2007: 3). La exclusión de las mujeres no era ni siquiera expresa, representando el colmo de la invisibilización. ¿Por qué, entonces, las mujeres no podían votar? Pues aunque la Ley no prohibía el sufragio expresamente, al estar el padrón electoral basado en el padrón militar (Ansaldi, 1999) y al excluir a las mujeres de las fuerzas armadas, la tácita prohibición se materializaba en silencio. Es importante revelar que las mujeres no aceptaban esta prohibición de manera sumisa:

"Si bien los derechos políticos crecieron rezagados respecto de otros derechos (especialmente los civiles y sociales), los años 20 encontraron un período de esplendor para el sufragismo local: se realizaron dos simulacros electorales en los cuales las representantes más conspicuas fueron candidatas. Además de las agrupaciones sufragistas y feministas y de las acciones de los partidos políticos -Alicia Moreau de Justo resulta una figura insoslayable como sufragista, feminista y socialista-, también se dieron experiencias fuera de los partidos, aunque fueron más excepcionales. La agrupación impulsada por Lanteri, el Partido Feminista Nacional (1918), único partido feminista con fines electoralistas de Argentina; y la experiencia multipartidaria de la Asociación Pro Derechos de la Mujer, presidida por Elvira Rawson (1918)" (Valobra, 2008:3).

Las mujeres comenzaban a cuestionar esta consideración de incapaces que les venía impuesta por las leyes patriarcales que necesitaban de su sumisión. Tal y como lo resalta Ansaldi (1999), la capitis diminutio de las mujeres era de por vida, como la de los dementes: son siempre súbditos, nunca sujetos de derechos. Tal y como lo expresa Carol Pateman (1995: 166): "Hasta bien avanzado el siglo XX, la posición legal y civil de la esposa se asemejaba a la del esclavo [...] un esclavo no tenía existencia legal separada de su amo, y un esposo y una esposa eran "una persona" la persona del marido".

Aunque el primer proyecto a favor del sufragio femenino fue presentado en 1919 por Rogelio Araya (Valobra, 2008), hubo que esperar hasta 1947 para que la demanda se convirtiera en Ley y las mujeres pudieran acceder a los derechos políticos. La sanción del sufragio femenino en Argentina se enmarca en una tercera ola de reconocimientos de los derechos políticos de las mujeres; ya que después de la Primera Guerra Mundial se reconoció el derecho al voto a las mujeres en Europa excepto en Francia (Perrot, 2008). Una segunda ola avanzó en tal reconocimiento entre los años 30 y 40 (Palermo, 2007), y la tercera se dio entre la Segunda Guerra Mundial y los años 60.

El que nos hayan excluido de la categoría de sujetos y de la categoría de individuos tiene que ver con el contrato sexual (Pateman, 1995) del que fuimos objeto las mujeres:

"El pacto originario es tanto un pacto sexual como un contrato social, es sexual en el sentido de que es patriarcal -es decir el contrato establece el derecho político de los varones sobre las mujeres- y también es sexual en el sentido de que también establece un 
orden de acceso de los varones al cuerpo de las mujeres [...]el contrato está lejos de oponerse al patriarcado, el contrato es el medio a través del cual el patriarcado moderno se instituye" (Pateman, 1995: 11).

De acuerdo a la teoría del contrato sexual, las mujeres no se constituyeron en sujetos de derecho al momento de la firma del contrato social, porque en virtud del contrato sexual, las mujeres fuimos objeto del mismo. Si la mujer es objeto, nunca puede ser sujeto, y como dice Pateman "la dominación de los varones sobre las mujeres y el derecho de los varones a disfrutar de un igual acceso sexual a las mujeres es uno de los puntos en la firma del pacto original. El contrato social es una historia de libertad, el contrato sexual es una historia de sujeción" (1995: 10).

Es en esta teoría donde encontramos la causa plausible que explica la exclusión de las mujeres del ámbito de los derechos. Casi emulando a la dialéctica del amo y el esclavo de Hegel, no se puede someter a un igual. Si las mujeres se convertían en ciudadanas, iba a ser más difícil mantenerlas en el plano del objeto, de ahí que se las excluyera.

Sin embargo, si considero que la ciudadanía femenina es un problema contemporáneo es porque hay consenso en que ser ciudadana es mucho más que tener habilitado el derecho al voto. Si defino la Democracia como un sistema político que "universaliza los derechos de los ciudadanos mediante la introducción de derechos sociales de repartición y derechos políticos de participación" (Greblo, 2002: 179) podemos pensar en una ciudadanía de doble carácter. No sólo la tradicionalmente planteada por Marshall e identificada como "pasiva", que postulaba que sin los derechos políticos y sociales, los derechos civiles eran de efectivo ejercicio sólo para una elite; sino en una ciudadanía activa que se comprometa en la participación política ${ }^{2}$. Es preciso aclarar que si las mujeres no politizábamos lo personal, difícilmente hubiéramos accedido a determinados derechos, ya que los derechos se conquistan.

¿Qué dimensiones tiene la ciudadanía en pleno siglo XXI para las mujeres en Argentina? Ya en 1947 fue conquistado el derecho al voto y en la década de los años 90 se logró una "ley de cupo" ${ }^{3}$ que garantiza un porcentaje de mujeres en las listas electorales. Tuvimos en Argentina una mujer presidenta por dos periodos consecutivos ${ }^{4}$, y reconocemos lideresas en el espectro político ${ }^{5}$ social. Sin embargo, considero que el hecho de no haber logrado el derecho

\footnotetext{
${ }^{2}$ Esta es originalmente una crítica realizada por la Nueva Derecha al concepto de derechos, pero aquí la reformulamos en una interpretación no conservadora, sino de resistencia: la participación política como resistencia y como la forma de articular las demandas del colectivo de mujeres. Véase Kymlicka y Norman (1997) y Marshall (1949).

${ }^{3}$ Ley $\mathrm{N}^{\circ} 24.012$ de 1991 por la que se modifica el código electoral y se establece como piso mínimo el $30 \%$ de candidatas mujeres en las listas electorales.

${ }^{4}$ La Presidenta Cristina Fernández de Kirchner gobernó en Argentina en los periodos 2007-2011 y 20112015 , en este último accedió al cargo con el $54.11 \%$ de los votos.

${ }^{5}$ En las últimas elecciones del año 2015, 5 de las 24 jurisdicciones lograron gobernadoras mujeres en las provincias de Buenos Aires, Santa Cruz, Catamarca, Tierra del Fuego y Santiago del Estero, es decir, un $20 \%$ de las provincias serán gobernadas por mujeres, lo cual es histórico e inédito en la historia de nuestro país.
} 
a la interrupción voluntaria del embarazo es la demostración cabal que todavía disputamos: la construcción de una ciudadanía plena que reconozca la propiedad sobre nuestros propios cuerpos.

\section{Estereotipos y ciudadanía femenina en los debates parlamentarios}

Los estereotipos de género, que forman parte del bagaje cultural en el marco de una organización social patriarcal, siempre juegan un papel que nos obtura a la consecución de la ciudadanía plena. Así sucedió en Argentina cuando se debatió en el parlamento el sufragio femenino en los años '30, y de igual manera sucedió en el año 2006, con oportunidad del debate parlamentario en torno a la Ley de anticoncepción quirúrgica.

El ser humano se concibe dicotómicamente. Su existencia se organiza en pares duales establecidos jerárquicamente y que sitúan a la mujer en el lado de lo débil -en contraposición a lo fuerte-, del lado de lo emocional -en contraposición a lo racional- y del lado de lo inferior -en contraposición a lo superior-:

"Siguiendo a Olsen (2009) podemos decir que la organización del mundo está dualizada y dichos dualismos, además de sexualizados, están jerarquizados. Esto significa que la comprensión del mundo en términos duales implica una o varias dicotomías: varón/mujer, razón/emoción, cultura/naturaleza, racional/irracional, objetivo/subjetivo; y que lo masculino se ha situado en un lugar del par dual que ostenta una posición jerárquica superior respecto del otro, donde se ha ubicado a las mujeres, tradicionalmente visto como lo negativo, lo inferior" (Cano, 2015: 11).

Esta interiorización construida culturalmente y sostenida teóricamente fue una de las razones por las cuales a las mujeres no se las consideraba aptas ni siquiera para opinar de temas políticos, y nótese que aun hoy muchas mujeres son descalificadas en ese terreno, sólo por el hecho de ser mujeres.

En los años '30 había un temor discursivo sobre el sufragio femenino y existía también un temor real. El temor discursivo podemos representarlo en los argumentos del Diputado Francisco Uriburu al momento de debatirse los derechos políticos de las mujeres en el parlamento. Como lo expresa Dora Barrancos, refiriéndose al Diputado: “dijo que otorgar el sufragio femenino es "fomentar la disolución de la familia con gérmenes de anarquía, es disminuir el poder marital ya socavado por la acción económica de la mujer, es propender a la disminución de los matrimonios [...] implicaba «la supresión del privilegio de su debilidad»"” (2007: 162). Podemos observar entonces, que se invocaba un "genuino" temor por la disolución de la familia como base de la sociedad, y por ende, se auguraba el caos. 
En cuanto al temor real, el fundamento de la exclusión de las mujeres de la arena política, se relaciona con el contrato sexual. En este contrato claramente se establece un varón superior sobre una mujer inferior. El contrato sexual otorga la prerrogativa, no sólo de acceder a los cuerpos de las mujeres, sino de definir su presente y su futuro como miembros de la sociedad: "mientras que para los socialistas, la ley del estado debía procurar liberar a las mujeres reconociéndoles sus derechos como individuos, para los conservadores la ley no podía avasallar los derechos privados y el orden sexualmente jerarquizado del mundo doméstico" (Palermo, 2007: 20).

La rígida división de lo público y lo privado, el primero de estos mundos reservado para los varones y el segundo asignado con exclusividad a las mujeres, se veía amenazado por las reivindicaciones feministas que abogaban por una igualdad, legal y real. La crítica feminista a la distinción dicotómica de lo público (relacionado con el mercado y el orden político) y de lo privado (relacionado con el ámbito familiar y domestico) se dirigió "tanto a la forma tradicional de trazar la frontera entre ambas esferas como al sentido mismo de la distinción [...] el ámbito público se transformaba en objeto de reflexión, teorización y legislación, mientras que la esfera domestica ... se trivializaba, aceptando como dato natural las relaciones patriarcales" (Rabotnikof, 1998: 9-10).

La consecuencia más remarcable de la sanción de una ley que permitiera a las mujeres elegir y ser elegidas en condiciones de igualdad con los varones, fue la posibilidad de trascender del ámbito familiar y doméstico como una realidad concreta. El acceso a la educación y el trabajo nos permitió a las mujeres cuestionar la jerarquización de los sexos dentro del sistema patriarcal (Valobra, 2010). En 1947, se sanciona la Ley 13.010 y se nos reconoce a las mujeres el derecho a elegir y ser elegidas en el espectro político, en el ámbito de lo público:

"La responsabilidad [de ir a votar], contrario al modo en que la habían pensado algunos legisladores, no se percibió como una extensión de sus roles familiares. Más bien, ellas se apropiaron del voto como una manera de conectarse con una entidad abstracta -la nación, la patria- y se deslindaron momentáneamente de la domesticidad, aunque sin abjurar de esos deberes" (Valobra, 2010: 74).

Que las mujeres accediéramos al sufragio fue la materialización de una demanda concreta, y tuvo la fuerza simbólica para desandar un camino estereotipado de la femineidad; aunque no se cuestionara el orden de género, ni la división sexual del trabajo, por parte de sus promotores:

"El peronismo a la par que afirmaba la igualdad en el campo espiritual, reforzaba la diferencia de roles que, en función de sus diferencias biológicas, hombres y mujeres debían cumplir en la sociedad. En este sentido, el peronismo retomó y fortaleció los planteos del maternalismo, al legitimar la participación política de la mujer como una extensión de su papel maternal” (Palermo, 2007: 24). 
Si la "naturalidad" de la mujer indicaba que su lugar estaba en la casa, el derecho a elegir y ser elegida en la arena política cuestionaba -a veces sin querer- esta ideología de la domesticidad. Aun cuando la Ley 13.010 se puede analizar desde una perspectiva estratégica para aumentar el caudal político (Palermo, 2007), lo cierto es que esta medida se tradujo en cambios considerables para la identidad femenina "[...] al cuestionar las jerarquías construidas sobre la base de las diferencias de género y al glorificar los atributos de la mujer de pueblo para la participación política" (Palermo, 2007: 29). Incluso, pensándolo desde la voz que les otorgaba la ley, para discutir cuestiones políticas en la mesa del hogar:

"La valorización de un mundo familiar politizado conllevaba además una igualación entre hombres y mujeres para expresar sus posiciones políticas [...] la obtención del derecho a elegir y ser elegida se correspondería con cambios similares en la vida del hogar. Así, Eva Perón recordaba a las mujeres que con el voto no sólo «defendemos el jornal mejor de tu marido y el acceso al techo digno y al pan común» sino que además «defendemos el derecho de opinar en la mesa del hogar que hicimos laboriosamente»" (Palermo, 2007: 26).

\section{El debate de 2006 en la Ley de anticoncepción quirúrgica: el significado de "poner el cuerpo"}

"Y no es casualidad que antes sólo se haya hablado de ligadura de trompas, porque durante muchos años esto ha tenido que ver con una cuestión simbólica donde lo que estaba en juego era el dominio del cuerpo de las mujeres...la concepción del cuerpo de las mujeres como un mero aparato reproductor, donde ellas eran guardianas pero no dueñas de sus hijos. En todo caso éramos víctimas de nuestro propio cuerpo, pero no dueñas"

Diputada Rodríguez (BA) Debate en la Cámara de Diputados el 28 de junio de 2006.

Esta Ley fue tratada en la Cámara de Diputados ${ }^{6}$ en el año 2006, haciendo uso de la palabra 21 diputadas y 15 diputados en un debate que fue arduo, y que terminó con 147 votos afirmativos, 41 negativos y 2 abstenciones de un total de 190 diputados/as presentes. En la Cámara Alta $^{7}$ tomaron la palabra 8 senadoras y 5 senadores, registrándose 35 votos por la afirmativa, 9 por la negativa y ninguna abstención. ${ }^{8}$

\footnotetext{
${ }^{6}$ Debate disponible en: Cámara de Diputados de la Nación. $208^{\circ}$ reunión $-138^{\circ}$ sesión ordinaria. Junio 28 de 2006. Periodo $\mathrm{N}^{\mathrm{o}} 124$.

${ }^{7}$ Debate disponible en su versión taquigráfica: Cámara de Senadores de la Nación. $18^{\circ}$ reunión - $16^{\circ}$ sesión ordinaria - 9 de agosto de 2006.

${ }^{8}$ Cabe explicitar que el análisis del debate parlamentario (que se dio en el año 2006) se realiza a través de los discursos de los/as congresistas y de los/as senadores/as del Parlamento constituido. En la Cámara de
} 
Como expuse previamente, el análisis en profundidad del debate parlamentario se vincula con la ciudadanía femenina, dado que todavía disputamos de la consideración de ser ciudadanas de pleno derecho. Cuando podamos decidir sobre nuestros cuerpos sin injerencias de las instituciones ni del colectivo de varones, podremos celebrar un paso más en el camino a la igualdad real. Considero que la Ley de anticoncepción quirúrgica de 2006 es un primer paso en ese sentido.

El trámite de este proyecto de Ley fue muy debatido por los y las legisladoras, incluso mucho más que cuando se trabaja sobre leyes que amplían derechos. No tuvo un tratamiento similar, por ejemplo, ni la Ley de Protección integral para prevenir, sancionar y erradicar las violencias contra las mujeres en los ámbitos en que desarrollen sus relaciones interpersonales, No 26.485 ni la Ley de Derechos de Padres e Hijos durante el Proceso de Nacimiento, No 25.929 conocida como "Ley de parto humanizado".. La diferencia fundamental se da en que en la Ley de anticoncepción quirúrgica lo que se debatía, en profundidad, es el dominio del propio cuerpo en relación con la sexualidad libre, separada de la reproducción que nos designa a las mujeres como envases de las generaciones siguientes.

A pesar de que esta Ley autoriza a que las mujeres podamos acceder a una ligadura tubaria y los varones acceder a la vasectomía en el sistema de salud público y privado, lo que en verdad se debatió, y así queda claro de la lectura de los discursos de los/as diputados/as y senadores/as, era el derecho de las mujeres de disponer sobre nuestros cuerpos -divorciando la sexualidad de la reproducción- ya que el foco se puso sobre el cuerpo femenino.

Hubo quienes alertaron a legisladores/as que consideraban que esta Ley no había tenido el debate social suficiente para ser sancionada ${ }^{9}$, así como también que hacía falta consultar a más expertas/os para poder tomar una decisión final. En seguida se les señaló que este es un debate que tiene historia en la sociedad argentina, demostrada por la cantidad de proyectos en el mismo sentido $^{10}$ que se presentaron desde la recuperación de la democracia en el año 1983:

"Este debate que viene desarrollándose en la sociedad desde hace más de 30 años seguramente proviene de una forma patriarcal y capitalista de concebir el cuerpo de la mujer como una propiedad social para preservar la especie y asegurar la distribución demográfica de la población, según intereses políticos y económicos, subordinándolas a mandatos que les confieren el carácter de ciudadanas” Diputado Galantini (Corrientes).

\footnotetext{
Diputados el tratamiento fue el día Junio 28 de 2006 en el Periodo de Sesiones No 124, y en la Cámara de Senadores el tratamiento de la Ley se dio con fecha del 9 de agosto de 2006.

${ }^{9}$ Por ejemplo la Diputada Jerez (Tucumán), que incluso pidió que el proyecto vuelva a Comisión para su tratamiento. También el Diputado Santander (La Rioja), el Diputado Vanossi (CABA) y a la Senadora González de Duhalde (BA) entre otros/as.

${ }^{10}$ Sólo en ese momento se estaba votando un proyecto de ley que sintetizaba otros que tenían estado parlamentario y que habían presentado la diputada Barbagelata, la diputada Bosch de Sartori, la Diputada Rico, la Diputada JI Marino y la Diputada GB Gutierrez.
} 
Para fundamentar la Ley, se resaltó el marco de derechos vigentes, la jerarquía constitucional de la $\mathrm{CEDAW}^{11}$ y su vinculación con el Programa nacional de salud sexual y procreación responsable ${ }^{12}$.

El argumento que primó en ambos recintos para justificar el tratamiento de la Ley fue el de justicia social, evidenciando que era una cuestión de clase y de recursos económicos acceder a determinadas prácticas prohibidas por una Ley del gobierno de facto del General Onganía ${ }^{13}$. Además, también se resaltó la "inequidad territorial", dado que había provincias que ya contaban con una norma en ese sentido, lo cual evidenciaba lo injusto que era que una mujer en Río Negro pudiera acceder a la práctica; y una de San Juan no.

El fundamento último del dictado de esta Ley fue la autonomía de la voluntad de mujeres y varones mayores de edad para disponer de su propio cuerpo, de acuerdo al ideario liberal que inspira nuestra Constitución y que se plasma en su artículo $19^{14}$. Varios/as legisladores/as se refirieron a que la primera propiedad de una persona es su propio cuerpo, lo cual se traducía en ciudadanía sexual. Como dije previamente, con la prohibición del aborto en nuestro país, nosotras las mujeres estaríamos aun disputando esta ciudadanía sexual.

A pesar de que los debates en ambas Cámaras giraron en torno a las mujeres, se insertó la perspectiva de género expresamente en los mismos a partir de manifestaciones que daban cuenta de la realidad que viven las mujeres debido a su adscripción genérica en el marco de una organización social patriarcal:

"Si bien el proyecto de Ley está pensado para hombres y mujeres, es indudable que éstas serán las más beneficiadas al momento de entrar en vigencia la normativa. La experiencia de las jurisdicciones que ya poseen legislación en este sentido, así como los pedidos judiciales para el acceso a las prácticas, demuestra un universo compuesto por una mayoría abrumadora de mujeres. A ello puede agregarse que resulta paradójico que un señor juez o una señora jueza pueda decidir sobre el cuerpo de cualquiera de nosotros" Diputada Rico (BA).

"Entonces, vamos a hablar de métodos anticonceptivos, porque las mujeres que están acá saben de qué estoy hablando, y los hombres, si no lo saben, lo van a aprender esta noche. Nosotras muy seguido, casi todo el tiempo, tenemos que ir al ginecólogo. Todas nosotras

${ }^{11}$ La Convención para Eliminar todas las formas de discriminación contra la mujer, conocida como CEDAW por sus siglas en inglés y firmada en el marco de Naciones Unidas en 1979, se integró como parte de nuestra Constitución Nacional en la última reforma constitucional en 1994.

${ }^{12}$ Aprobado por ley $\mathrm{N}^{\circ} 25.673$ en el año 2003.

${ }^{13}$ En el año 1966 se produjo un golpe de Estado en nuestro país, que ubicó al General Onganía en la presidencia hasta 1970, derrocando al Presidente Arturo Illia que había sido elegido por mandato popular.

${ }^{14} \mathrm{El}$ artículo $19 \mathrm{CN}$ dice: "Las acciones privadas de los hombres que de ningún modo ofendan al orden y a la moral pública, ni perjudiquen a un tercero, están sólo reservadas a Dios, y exentas de la autoridad de los magistrados. Ningún habitante de la Nación será obligado a hacer lo que no manda la ley, ni privado de lo que ella no prohíbe". 
tenemos que ir porque tomamos pastillas que nos hacen mal, nos hacen retener líquido, nos ponen gordas, no hacen mal al hígado, vomitar. Sino, tenemos que recurrir al DIU, que no es anticonceptivo sino abortivo. Se encarna, trae menstruaciones dolorosas, profusas, se corre, trae miles de problemas [...] verdaderamente nosotras no somos animales que tenemos sexo para reproducirnos nada más. Queremos disfrutarlo, como dijo la diputada preopinante” Diputada Pérez (BA).

“¿Cuántas mujeres a veces tienen que hacer el amor obligadas, cuando por una decisión personal el hombre no utiliza preservativo? Es la mujer la que sufre los desórdenes hormonales cuando toma una pastilla, es la mujer la que soporta las practicas invasoras que en general presuponen los controles periódicos ginecológicos o la colocación, el acomodamiento o el retiro de un DIU, es la mujer la que sufre la deformación de su cuerpo en el embarazo, es la mujer la que soporta la cesarea. Es decir que la mujer sufre todo eso para engendrar o para cuidarse” Diputado Rossi (Santa Fe).

Entre los argumentos en contra, debo decir que el ejercicio de la imaginación que se plasma en los debates parlamentarios es digno de elogiarse. En principio, algunos/as de los/as legisladores objetaron la norma por miedo a un "vendaval de ligaduras tubarias", utilizando los mismos argumentos que se utilizaron en el año 1987 para oponerse a la sanción del divorcio vincular. Resultan curiosas las denuncias que se hicieron a quienes estaban promoviendo la discusión en el recinto:

"Disculpen esta autorreferencia, pero quiero contarles las cosas que pasamos para lograr esa ley [en la provincia de La Pampa]. En realidad, encontrábamos algún sector de la sociedad que nos decía que éramos favorecedores de la prostitución, que con esta ley ibamos a extender la promiscuidad a toda la sociedad, que iba a aparecer el rebrote de las enfermedades de transmisión sexual, que en realidad estábamos atentando contra la formación de las familias, que la gente iba a dejar de tener hijos, que las familias se iban a disgregar, argumento que ya habían utilizado cuando se trató la ley de divorcio. En verdad, quiero decirles que estas últimas semanas hemos asistido, casi con estupor, a las mismas - absolutamente las mismas - argumentaciones que hace diez años atrás" Senadora Gallego (La Pampa).

Parte de los/as legisladores/as consideraban que ligarse las trompas o realizarse una vasectomía puede ser objeto de moda que curiosamente adoptaría la juventud, todo ello sin referirse a ninguna estadística que pudiera sustentar dicha afirmación. La "preocupación" acerca de que los/as jóvenes accedan a estas prácticas indiscriminadamente, demostró no sólo que no consideran a la juventud como seres completos, sino dignos de tutela, -al igual que se considera así a los/as pobres- como también un desconocimiento profundo de en qué circunstancias las 
mujeres se acercan a solicitar esta práctica. Al respecto, la diputada por Santa Fe, la Sra. Augsburger comentó que en Rosario (ciudad donde ya existía la Ley para ese entonces) el perfil de las solicitantes era de más de 39 años y con más de 4 hijos, demostrando así que la disponibilidad de estadísticas (una deuda pendiente en Argentina en relación a las violaciones de los derechos de las mujeres) ayuda a dejar de fantasear con suposiciones o datos inventados que sólo confunden a la ciudadanía. La diputada Hernández por Río Negro hizo lo propio. Asimismo lo aclaró la Senadora Gallego por La Pampa:

"Brevemente, me permitiré leer algunas pequeñas estadísticas correspondientes a estos casi tres años de aplicación de la ley en nuestra provincia. Al amparo de esta ley se han hecho 415 ligaduras tubarias, y aproximadamente ocho vasectomias -los hombres siguen siendo renuentes a controlar per se la descendencia; vamos a mejorar eso, pero todavía sigue siendo responsabilidad mayoritaria de la mujeres la definición de este tipo de cosas. De las encuestas prequirúrgicas que se hicieron en virtud de nuestra ley surgió que el 50 por ciento de las mujeres que se realizaron la ligadura de trompas vivían en estado de concubinato. De estas 415 mujeres, el 75 por ciento tenía educación primaria completa, el 3 por ciento - un porcentaje mínimo - primaria incompleta, y el resto secundaria completa y, en algunos casos, universitaria. Mayoritariamente, el método que utilizaban hasta ese momento era el condón. Y cuando se les preguntó por qué motivo querían hacerse esa práctica, la respuesta categórica fue que no querían tener más hijos. No plantearon una cuestión social ni ningún otro aspecto que ande sobrevolando por ahi; la decisión autónoma de esas mujeres fue no querer tener más hijos [...] En cuanto al número de embarazos previos - para los que dicen que a partir de ahora cualquiera en cualquier momento podrá operarse, y que una niña de 18 años pedirá la ligadura de trompas y se la harán-, el promedio dio 5,4” Senadora Gallego (La Pampa).

Además, la oposición al proyecto señaló que la utilización de este método, definido como esterilizante $^{15}$, castrativo, e incluso como una "mutilación”, y como "injuria quirúrgica" traería aparejado un alza en la tasa de enfermedades infectocontagiosas -porque aparentemente las personas dejarían de usar preservativo para prevenir dichas enfermedades-.

Considero que el paternalismo sobre las mujeres pobres que fue la "frutillita de esta torta" ${ }^{16}$ de argumentos patriarcales disfrazados de conciencia nacional y de solidaridad de clase:

"Aunque suene feo voy a decirlo con todas las letras: ¿acaso está dirigida [la Ley] a los cabecitas negras ${ }^{17}$ ? Si apunta a los sectores más humildes y más desprotegidos de la

\footnotetext{
${ }^{15}$ Fue la Diputada Gutiérrez (Santa Cruz) quién explicó a la audiencia que la anticoncepción quirúrgica no es esterilizante, ya que no afecta a los óvulos ni a los espermatozoides, sino que simplemente impide su unión. Es decir que podría lograrse un embarazo, por ejemplo, con la técnica de fertilización in vitro aún en varones y mujeres intervenidos quirúrgicamente.

${ }^{16}$ Expresión que significa cuestión que se acaba o se remata.
} 
sociedad, también estamos hablando de los sectores con menor nivel educativo y menores posibilidades de acceso a la educación [...] Aprobar la utilización de una herramienta que no sabemos cómo va a ser usada, tanto por parte del personal médico como de la ciudadanía desinformada y no educada en esta cuestión, me parece que no favorece la posibilidad de expansión del hombre en el universo y del universo mismo [sic]" Diputado Lusquiños (San Luis).

La respuesta, en voces femeninas, no se hizo esperar:

"La gente nos vota para implementar políticas públicas, defender y garantizar derechos ¿Por qué cualquiera de nosotros va a saber más que una persona individual qué es lo que le conviene para su propio plan de vida? [interrumpida por aplausos] Sinceramente, no siento que en lo personal tenga más competencia que ninguna de las personas que me votaron para saber qué es lo que tiene que hacer con su sexualidad o cómo decidir el número de hijos...ni ninguna orea cuestión de su vida privada [...] nadie me votó para eso” Diputada Rodríguez (BA).

"Esta concepción tutelar de la pobreza fue la misma que en su momento —así lo dijo el senador Giustiniani- se utilizó para restringir el voto de los pobres, analfabetos y de las mujeres. Es decir, siempre se usa el mismo criterio. Hay unos poquitos que pueden decidir qué es mucho mejor para los muchos. Cuando los poquitos empiezan a decidir sobre la vida y los derechos personalísimos de los muchos comienzan los Estados interventores, totalitarios e inmorales" Senadora Ibarra (CABA).

El arrepentimiento también fue causa de debate, donde subyacían argumentos sobre que tener hijos/as es el fin último de las mujeres. A esto la Diputada Rodríguez contestó con solvencia y sin paternalismo: "[...] también tendrán derecho a una decisión irreversible [...] ¿Qué otra decisión más irreversible hay en la vida -para mi ninguna-que tener un hijo?”.

Se denuncia fuertemente que esta Ley estaría en connivencia con las políticas demográficas de las potencias imperialistas para reducir las poblaciones del "tercer mundo" que amenazarían el bienestar de los/as ciudadanos/as del primer mundo, hechas manifiestas en el Informe Kissinger de la década del '70 y que seguirían vigentes en instrumentos como el que se trajo a debate. La respuesta al "gobernar es poblar" fue que no podía hacerse a expensas de los cuerpos de las mujeres. Incluso se comparó la Ley con una ley del periodo hitleriano ${ }^{18}$ :

\footnotetext{
${ }^{17}$ Forma despectiva de dirigirse a las personas de los sectores populares que viene desde la mitad del siglo $\mathrm{XX}$ cuando, durante en el transcurso del primer gobierno peronista, llegaron a la ciudad muchos migrantes internos -de las provincias argentinas-, que eran considerados pobres - por lo tanto indignos de la ciudad capital- y además estigmatizados por su color de piel.

${ }^{18}$ Cortesía del Diputado Lusquiños (San Luis).
} 
"Muchos señores diputados han justificado este proyecto desde la equidad social y la igualdad de oportunidades. Al respecto quiero decir varias cosas. Esta bandera de la opción por los pobres, que suelen enarbolar algunos, a veces esconde una solución solapada: disminuir la pobreza reduciendo a los pobres, aplicando una política antinatalista forzada, siempre solventada por los solícitos y solidarios organismos de préstamos internacionales. Pareciera que para que haya menos pobres vamos a hacer que nazcan menos niños en vez de generar las condiciones para que puedan vivir dignamente incluso los que ya nacieron” Diputada Jerez (Tucumán).

Y no faltó quien defendiera los intereses del cuerpo médico y de las instituciones privadas (promoviendo el derecho a la "objeción de conciencia institucional") ${ }^{19}$, e incluso la necesidad de que todas las instituciones posibles se pronuncien acerca de la conveniencia de que las mujeres decidamos sobre nuestro propio cuerpo:

"Este es un método de planificación familiar. Esta norma -y lo digo con toda humildadtiene mucho de autoritaria y de soberbia ... tiene escasa jerarquía legislativa, y ha dejado a los médicos indefensos, puesto no se ha consultado a ninguna academia profesional", Diputado Santander (La Rioja).

"Si ellos [los medios de comunicación] se hubieran ocupado de esto se podría haber generalizado el debate con la participación de todas las organizaciones no gubernamentales, de las academias nacionales y de las facultades que han tenido y siguen teniendo algo que decir porque les concierne esta cuestión” Diputado Vanossi (CABA).

"Según publicaciones de la ciencia médica, la esterilización anticonceptiva es la mutilación del propio cuerpo mediante la destrucción deliberada de una importantísima facultad natural: la fertilidad [...] Dónde deja este proyecto la opinión valiosa del médico? ¿Dónde deja la opinión de un psicólogo y hasta la de una asistente social?” Diputada Mansur (BA)

La referencia al derecho del cuerpo médico a opinar es de resaltar. Tradicionalmente el cuerpo femenino fue gobernado, o bien por los maridos, o bien por los médicos. A las mujeres se les sustrajo los conocimientos sobre su propio cuerpo y sobre la reproducción en la edad media (Federici, 2010), persiguiendo como brujas a las mujeres parteras que trasmitían estos saberes, parte de lo que Federici denomina acumulación originaria precapitalista. Desde allí que la autoridad se trasladó al médico (en ese entonces varón) quien se ubicó como la voz de

${ }^{19}$ Cortesía de la Senadora Negre de Alonso (San Luis). 
autoridad en relación con el cuerpo femenino, infantilizando a las mujeres desde un paternalismo autoritario que establece que ellos saben más que nosotras sobre lo que pasa en nuestro cuerpo.

"Dicho de otro modo, la sexualidad y el placer «femeninos» se construyen en el espacio de tensión y de encuentro de al menos dos instituciones: la institución matrimonial heterosexual, en la que las mujeres están sujetas a sus maridos, y las instituciones médicas, en las que las mujeres están sujetas a la jerarquía clínica como pacientes" (Preciado, 2002: 91).

El último de los argumentos patriarcales por excelencia expuestos en el recinto, fue la vulneración de los derechos del cónyuge ante una decisión unilateral por parte de la mujer. Al respecto rescato la respuesta de la senadora Ibarra:

“El consentimiento del cónyuge es otro de los temas planteados acá que yo también quiero abordar. Me pregunto si al hablar de consentimiento del cónyuge estamos hablando de la propia salud, del propio cuerpo. ¿Alguien cree que en una pareja bien avenida, que discute, que tiene relaciones sexuales cuando los dos lo desean, que eligen y planifican su vida, un día la mujer dispara y se va a ligar las trompas? Esto es una opción. Lo dijo muy bien ayer la senadora Alicia Kirchner. Esta es una opción. La verdad es que en las familias bien avenidas, aquellas donde hay diálogos existe respeto por la integridad del otro, por la dignidad, por el deseo o el no deseo, el problema no es este; realmente no lo es. Se discute en las parejas cuando se busca un hijo y se lo recibe con alegría. Acá estamos dando la opción, el derecho a quien no le preguntan, a quien no tiene cómo, a quien está en riesgo y tiene derecho a decidir y a cuidarse. Pero parece que acá escandaliza a alguna gente cuando la mujer sale a defenderse y a cuidarse. No escandalizan los 500 mil abortos clandestinos por año, donde se van las vidas de las mujeres. Escandaliza cuando la mujer se defiende. Además, esto viene de los mismos sectores con las mismas convicciones con los que tenemos que discutir. Se habla de educación y después, cuando nos referimos a la educación para la sexualidad, se viene con el problema de que no hay que repartir preservativos; de que los métodos anticonceptivos, no; $y$ algunos no terminan de discutir el tema confesional en la educación. Siempre nos encontramos con los mismos criterios que llevan a la mujer a arrinconarse en el peor de los mundos" Senadora Ibarra (CABA).

Aunque el debate de esta Ley está plagado de argumentos feministas que se esgrimieron para responder a aquellos argumentos patriarcales y conservadores que intentaban disfrazarse de preocupación por los/as jóvenes, por los/as pobres y por los cónyuges, emerge por fin el 
argumento de que esta Ley trata del derecho a poder disfrutar de la sexualidad, tanto por parte de los varones (quienes tradicionalmente la disfrutan) como fundamentalmente por parte de las mujeres, y esa es la razón última de tantas voces en contra de la sanción de la Ley:

"Se han olvidado [los/as diputados/as] de que esa mujer todas las noches va a su casa y quiere tener placer. Desean tener placer sexual y no verse obligadas a decir que sufren un problema coronario o de presión arterial severa, lo que les impide tomar anticonceptivos. ¡Yo también quiero tener placer sexual, aunque tenga 45 años! ¡Necesito una ligadura de trompas y tengo que ir a preguntarle a un juez si me autoriza! Esas mujeres dicen que son personas como cualquier otra. Aunque tengan 10 hijos, una enfermedad severa o estén solas, deben tener la posibilidad de esta intervención quirúrgica” Diputada Bosch de Sartori (Misiones).

Que las mujeres recuperemos el dominio del cuerpo y que podamos elegir libremente el número de hijos/as que queremos tener, o si queremos o no tenerlos, es una preocupación para el patriarcado ${ }^{20}$, dado que esta es una de las formas que tiene de controlar a las mujeres -a través del control de sus cuerpos y de su fuerza de trabajo-.

Celebro que en el debate por esta Ley haya emergido la sororidad ${ }^{21}$, reconociendo la desigualdad que existe entre las propias mujeres. La sororidad "emerge como alternativa a la política que impide a las mujeres la identificación positiva de género, el reconocimiento, la agregación en sintonía y la alianza" (Lagarde, 2014: 559). Es decir, que se trata no sólo de ser honestas para visibilizar las violencias y discriminaciones que sufrimos a diario por el hecho de ser mujeres en todos los estratos sociales, sino también de solidarizarse con aquellas que además, tienen en sus cuerpos, otras marcas de subalternidad:

"Aun cuando ninguna de nosotras hagamos uso del derecho de hacernos una ligadura de trompas, debemos garantizar el ejercicio de este derecho a aquella cuya única posibilidad es ésta por razones de salud, no por prescripción médica, que son cosas bien distintas [...] la salud es mucho más que un útero y mucho más que un ovario. La salud es la cabeza de esa mujer que puede soportar tener o no tener más hijos [...] ¿Quién más que una mujer puede decidir qué hacer con su propio cuerpo cuando su decisión no afecta a terceros?" Diputada Ríos (Tierra del Fuego).

\footnotetext{
${ }^{20}$ De acuerdo a Heidi Hartmann, "La base material sobre la que se asienta el patriarcado estriba fundamentalmente en el control del hombre sobre la fuerza de trabajo de la mujer. El hombre mantiene este control excluyendo a la mujer del acceso a algunos recursos productivos esenciales (en las sociedades capitalistas, por ejemplo, los trabajos bien pagados) y restringiendo la sexualidad de la mujer" (Hartmann, 1980:97).

${ }^{21}$ Sororidad del latín soror, sororis, hermana, e-idad, relativo a, calidad de... (Lagarde, 2014:560).
} 
"No creamos que todas las personas pueden vivir situaciones de relación de pareja o de relaciones sexuales responsables: hay infinidad de personas que son víctimas de violencia, del alcoholismo y de otras situaciones que no les permite tener métodos adecuados para regir su fertilidad. Esta es una realidad. El que crea que "porque yo lo pude hacer", "todo el mundo lo puede hacer", se equivoca. La triste realidad es que hay cientos de miles de mujeres que no lo pueden hacer" Senadora Gallego (La Pampa).

\section{Reflexiones finales}

A lo largo de este trabajo se intentó poner de relieve el nivel de discusiones políticas que se dan en torno a la ciudadanía de las mujeres, partiendo de la base de que aún no somos consideradas sujetos de derecho plenas, desde que no tenemos total dominio de nuestros cuerpos. La soberanía aún la detenta el Estado, las Iglesias y el colectivo de varones. La propiedad del cuerpo, es una cuestión política.

Recorrimos la historia de los derechos políticos de las mujeres en Argentina para dar cuenta de que la ciudadanía no se define sólo por el hecho de votar o ser elegidas, aunque ese fue un primer paso para poder profundizar en la discusión. La ciudadanía se manifiesta en el control y en la propiedad sobre nuestro propio cuerpo. Y la Ley de anticoncepción quirúrgica fue un primer paso en ese sentido, un nuevo punto de partida para llevar adelante la discusión sobre el derecho al aborto.

Cada vez que se tratan temas que competen directamente a la sexualidad y libertad de las mujeres, el debate es álgido. Así sucedió en 1987 cuando se debatió sobre el divorcio vincular, cuando se debatió la Ley de educación sexual integral (Ley $\mathrm{N}^{\circ} 26.150$ de 2006) y en esta ocasión al debatirse la Ley de anticoncepción quirúrgica. Aunque el texto de la Ley contempla los derechos de mujeres y varones, lo cierto es que el debate estuvo centrado en los cuerpos de las mujeres, dado que tradicionalmente siempre hubo otros que decidieron sobre los mismos.

Que este tema haya llegado al recinto parlamentario está íntimamente relacionado con que haya mujeres ocupando esos espacios, y mujeres feministas impulsando y representando las luchas del colectivo de mujeres. Aunque coincido con Dora Barrancos cuando expresa que:

"Conformar un cuerpo de mujer no asegura automáticamente una subjetividad capaz de oponerse a las jerarquías de género. Tener cuerpo de mujer no significa impregnarse de una agenda feminista, de la misma manera que pertenecer a un estrato social dominado no equivale a manifestar adecuadamente sentimientos rebeldes" (Barrancos, 2008: 25)

Porque de hecho muchas veces las mujeres somos férreas guardianas del patriarcado. No obstante, lo cierto es que fue la presencia en el recinto de las mujeres, utilizando los argumentos 
feministas, lo que logró desnudar el meollo del asunto, y decir claramente sobre qué se estaba debatiendo. Una oportunidad que no tuvimos en la década de los '30 cuando los senadores debatieron acerca de los derechos políticos de las mujeres, excluyéndonos del recinto por otros 15 años más.

En el debate acerca del cuerpo, vuelve a emerger el contrato sexual del que aun somos objeto (Pateman, 1995), y así surgió en el recinto parlamentario cuando por ejemplo se refirieron los derechos de los cónyuges sobre el cuerpo de las mujeres, o incluso los derechos del cuerpo médico sobre el mismo cuerpo. Y todo ello sin mencionar la tutela que se ejerce con más ahínco sobre las mujeres jóvenes y las mujeres pobres, ambas consideradas, desde una visión adultocéntrica y clasista, como seres aún más incompletos.

Tampoco sorprende que la oposición al proyecto no haya reflexionado sobre cómo las mujeres les ponemos el cuerpo a los proyectos nacionales. Pensar que "gobernar es poblar" y que ello debe hacerse a expensas de los vientres femeninos. Es otro más de los argumentos patriarcales, que disfrazados de "interés nacional", siguen sometiendo a las mujeres en un régimen de dominación.

Quisiera cerrar el presente artículo, diciendo que aunque esta Ley lleva 10 años sancionada, podemos pensar que en el caso de debatirse en algún momento el derecho al aborto, los argumentos a favor serán los mismos, centrados en la autonomía de la voluntad de las mujeres, y referidos a la justicia social que evidencia que quien puede pagarlo puede acceder hoy a esta práctica, y son las mujeres pobres las que pagan con su vida la clandestinidad. Sin embargo, no creo que los argumentos en contra sean los mismos, sino que los que se esgrimieron en el debate por esta Ley será un piso argumental para desarrollar un potencial argumentativo que seguramente sorprende a la más preparada.

Por último, quiero resaltar que en el debate por esta Ley, varios diputados/as y senadores/as "valientemente" se refirieron a la necesidad de debatir el asunto del aborto en el parlamento (pedido que lleva mucho más que 10 años ignorándose) y de esos aportes, rescato el siguiente para cerrar el presente:

“¿De qué estamos hablando acá? Estamos hablando de la decisión de personas adultas sobre su plan de vida, respecto de su comportamiento sexual, de su capacidad reproductiva, donde el Estado no tiene injerencia. Las políticas positivas a favor de la familia deben tratar de realizarlas, pero no deben invadir la esfera privada y mucho menos a costa de las mujeres, que luego son las que aportan los pavorosos números de 500 mil o 600 mil abortos anuales con más de mil mujeres muertas por año a raíz de esta práctica y donde conocemos las situaciones sociales que fueron explicadas y sobre las que no voy a insistir nuevamente [...] Parece que la gente nos hubiera votado para tutelar sobre aquellos que pensamos que no están en iguales condiciones que alguna élite que sí sabe qué es lo mejor para sus vidas" Senadora Ibarra (CABA). 
Actualmente, el debate por el derecho al aborto sigue pendiente, siendo considerada una de las deudas más profundas de la democracia (retornada en 1983) con el colectivo de mujeres. A pesar de que el movimiento feminista local ha construido una demanda en torno a esta cuestión, ésta aún no se ha convertido en una cuestión estatal ni se ha abordado en el Parlamento argentino. La disputa por el control del cuerpo de las mujeres sigue vigente y la construcción de una ciudadanía sexual sigue pendiente.

\section{BIBLIOGRAFÍA}

- Ansaldi, Waldo (1999): “Crear el sufragante: la universalización masculina de la ciudadanía política en argentina. La reforma electoral de 1912” en Anales, Nueva Época, 2, Suecia, [en línea] Disponible en http://www.catedras.fsoc.uba.ar/udishal/art/crearsufragante.pdf (recuperado el 28/02/2016).

- Barrancos, Dora (2007): Mujeres en la sociedad argentina. Una historia de cinco siglos. Buenos Aires: Sudamericana. . (2008) Mujeres entre la casa y la plaza. Buenos Aires: Prometeo.

- Cano Callejo, Julieta (2015): "Perspectiva de género en las sentencias argentinas: ¿Una herramienta de lucha contra el patriarcado?". Trabajo Fin de Máster Universitario en Investigación Aplicada en Estudios Feministas, de Género y Ciudadanía (Plan de 2013). Codi: SBH023. Curso: 2014-2015 [en línea] Disponible en el Repositorio de la Universitat Jaume I: http://repositori.uji.es/xmlui/handle/10234/142652 [28/02/2016].

- Federici, Silvia (2010): Calibán y la bruja. Mujeres, cuerpo y acumulación originaria. Buenos Aires: Tinta Limón.

- Giordano, Verónica (2012): “Instantáneas del camino de los derechos políticos femeninos en la construcción del orden en América Latina" en Revista Ciencias Sociales, $\mathrm{n}^{\circ}$ 82, [en línea] Disponible en: http://www.sociales.uba.ar/wp-content/uploads/sociales_82.pdf [28/02/2016].

- Greblo, Edoardo (2002): Democracia. Léxico de política. $1^{\circ}$ Ed. Buenos Aires: Nueva Visión.

- Hartmann, Heidi (1980): “Un matrimonio mal avenido, hacia una unión más progresiva entre feminismo y marxismo", Zona Abierta, 24. p.85-113.

- Hunter, Rosemary et al. (2010): "Feminist Judgments: An Introduction" En Hunter, Rosemary et al. (Eds.). Feminist Judgements. From Theory to Practice. Orford and Portland, Orengon: Hard Publishing.

- Jelin, Elizabeth (1996): "Las mujeres y la cultura ciudadana en América Latina", Trabajo preparado dentro del programa Women in the service of civil peace de la División de Cultura, UNESCO, [en línea] Disponible en http://www.equidad.org.mx/index.php/es/2015-06-01-2140-21/ciudadania, [12/07/2015].

- Kymlicka, Will y Norman, Wayne (1997): "El retorno del ciudadano. Una revisión de la producción reciente en teoría de la ciudadanía". En: Ágora, no. 7, pp. 5-42.

- Lagarde y de los Ríos, Marcela (2014): El feminismo en mi vida: hitos, claves, y topías. 
México: Horas y Horas.

- Marshall, Thomas Humphrey (1949): “Ciudadanía y clase social”. En: Conferencias en Cambridge, [en línea] disponible en www.catedras.fsoc.uba.ar/isuani/marshall.pdf [20/06/2015]

- Olsen, Frances (2009): "El sexo del derecho". En Ávila Santamaría, R., Salgado, J. y Valladares, L. (Comps.) (2009). El género en el derecho. Ensayos críticos. Ecuador: Ministerio de Justicia y Derechos Humanos.

- Palermo, Silvana (2007): "Quiera el hombre votar, quiera la mujer votar. Género y ciudadanía política en Argentina (1912-1947), [en línea] Disponible en http://historiapolitica.com/datos/biblioteca/palermo.pdf [28/02/2016].

- Pateman, Carole (1995): El Contrato Sexual. México: Anthopos/UAM.

- Perrot, Michelle (2008): Mi historia de las mujeres. Argentina: Fondo de Cultura Económica.

- Preciado, Beatriz (2009): "Terror anal: Apuntes sobre los primeros días de la revolución sexual" en Hocquenghem, Guy. El deseo homosexual. Barcelona: Melusina.

- Rabotnikof, Nora (1998): "Público-Privado". En Debate feminista, 18, p. 3-13.

- Reverter Bañón, Sonia (2008): “Sociedad civil, ciudadanía y género”, en: Aljaba [online], vol.12, pp.33-52.

- Smart, Carol (2000): "La teoría feminista y el discurso jurídico" En Birgin, Haydée (Comp.) El derecho en el género y el género en el derecho. Buenos Aires: Biblós.

- Valobra, Adriana (2008): "Feminismo, sufragismo y mujeres en los partidos políticos en la Argentina de la primera mitad del siglo XX" en @mnis, Revue de civilisation contemporaine de l'université de Bretagne Occidentale (UBO, Brest), [en línea] disponible en http://amnis.revues.org/666 [28/02/2016].

. (2010): Del hogar a las urnas. Recorridos de la ciudadanía política femenina. Argentina, 1946-1955. Rosario: Prohistoria, [en línea] Disponible en http://es.scribd.com/doc/34053743/DEL-HOGAR-A-LAS-URNAS [28/02/2016]. 